\title{
ORGANIZACIÓN DE LA INFORMACIÓN EN SITIOS WEB PERIODÍSTICOS
}

\author{
Misleiny Acosta-Valdés, Zulia Ramírez-Céspedes y Liliam Marrero-Santana
}
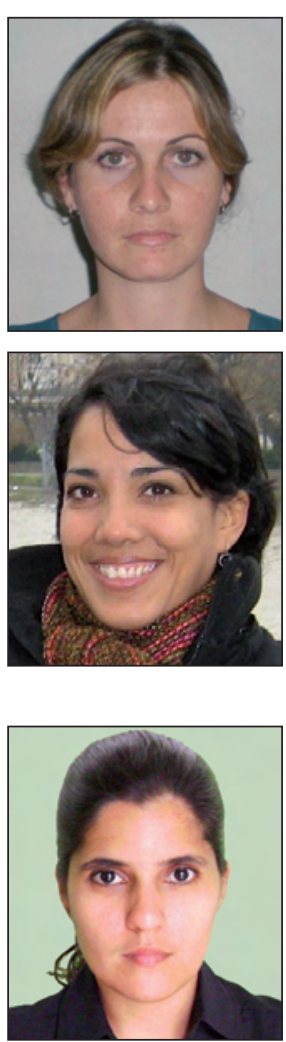

\section{Resumen}

Con los sistemas colaborativos y de participación, los usuarios de la Web crean y difunden contenidos mediante blogs, wikis y redes sociales. De esta forma emisor y receptor intercambian roles, transformando el proceso de generación de información que necesita ser estructurada y organizada eficientemente. Se presentan los resultados de un estudio de benchmarking sin carácter evaluativo aplicado a seis webs de prensa, para determinar las tendencias más recientes en cuanto a organización de la información, navegación, etiquetado, búsqueda, utilización de elementos multimedia, participación y alfabetización en el ejercicio del periodismo digital. El análisis se auxilia de una guía de benchmarking elaborada a partir de diferentes propuestas evaluativas.

\section{Palabras clave}

Periodismo digital, Organización de la información, Benchmarking, Arquitectura de la información.
Misleiny Acosta-Valdés es licenciada en bibliotecología y ciencia de la información por la Universidad de La Habana (2010). Becaria del Departamento de Ciencias de la Información en la Facultad de Comunicación de la Universidad de la Habana.

\author{
Universidad de La Habana. Facultad de Comunicación \\ Avenida de los Presidentes, n. 506, entre 21 y 23, vedado \\ 10400 Ciudad de La Habana, Cuba \\ misleiny.acosta@fcom.uh.cu
}

Zulia Ramírez-Céspedes, licenciada y máster en bibliotecología y ciencia de la información por la Univ. de La Habana, es profesora del Depto. de Ciencias de la Información en la Fac. de Comunicación de dicha universidad, con docencia en las asignaturas de arquitectura de información y recuperación de información. Es doctoranda en documentación e información científica en la Univ. de Granada.

Universidad de La Habana. Facultad de Comunicación Avenida de los Presidentes, n. 506, entre 21 y 23, vedado 10400 Ciudad de La Habana, Cuba zulia@fcom.uh.cu

Liliam Marrero-Santana, licenciada en periodismo por la Universidad de La Habana (2007), es profesora del Departamento de Periodismo en la Facultad de Comunicación de la Universidad de la Habana, con docencia en la asignatura de periodismo hipermedia. Es maestranda en ciencias de la información.

\section{Title: Information organization on newspaper web sites}

\begin{abstract}
With the development of collaborative systems, the web users create and disseminate information across the blogs, wikis and social networks. In that dynamic process, transmitter and receiver change roles to generate a huge amount of information that needs to be organized efficiently. We present the results of a benchmarking study, the goal of which is to determine the most recent tendencies for information organizing, navigation, searching and participation in six of the most significant newspaper sites.
\end{abstract}

\section{Keywords}

Online journalism, Information organization, Benchmarking, Information architecture, Digital newspapers. 
Acosta-Valdés, Mileiny; Ramírez-Céspedes, Zulia; Marrero-Santana, Liliam. "Organización de la información en sitios web periodísticos". El profesional de la información, 2011, enero-febrero, v. 20, n. 1, pp. 54-60.

DOI: 10.3145/epi.2011.ene.07

\section{Introducción}

El paso del periodismo al entorno digital ha dado lugar a la aparición de un nuevo medio de comunicación con otras maneras de implementar las prácticas en la profesión periodística, sus contenidos y sus relaciones con el público: el lector tradicional pasa a ser un usuario con identidad, que interactúa y participa dinámicamente.

Los rasgos esenciales de la comunicación en red -hipertextualidad, multimedialidad e interactividad-, en la medida en que han evolucionado los modelos de desarrollo del periodismo digital, configuran un discurso periodístico que renueva las concepciones tradicionales de los géneros y, en opinión de varios expertos, favorece un nuevo periodismo propio de la Red.

Rodríguez-Martínez, Codina y Pedraza-Jiménez (2010) plantean que "entre los contenidos que pueden encontrarse en la Web, las noticias ocupan un lugar cada vez más destacado", y que "los sitios web de los medios han aumentado progresivamente la cantidad de contenidos, al tiempo que los han hecho más atractivos".

Con el fin de valorar si la organización de la información de los principales periódicos digitales cubanos está en correspondencia con las recientes tendencias de organización, navegación, búsqueda y participación en la prensa digital, se decidió realizar un estudio de benchmarking cualitativo de varios sitios web periodísticos reconocidos internacionalmente.

\section{Estudios comparativos o benchmarking}

Tenemos el propósito de identificar la forma en que organizan y estructuran la información algunos medios digitales, así como la representan y qué posibilidades de navegación y recuperación presentan.

Según Martínez-Priego (2006) el benchmarking es una técnica de análisis usada en general para evaluar y comparar un producto o servicio con los demás que son similares. Utilizada en el entorno digital, "es el estudio de estructuras web de la competencia del cliente, o equivalentes en términos de contenidos y orientación, con el objetivo de poner el proyecto en su justo contexto y saber con qué requisitos mínimos debe contar para 'entrar a la cancha' con posibilidades de proporcionar una alternativa de servicios y soluciones para los usuarios que constituyen el público objetivo".

La evaluación de productos similares o análisis de la competencia, "es un proceso importante dentro de los proyectos de arquitectura de la información (IA), dado que su realización permite identificar debilidades y fortalezas de sitios web semejantes al nuestro. Esto nos ayuda a incorporar los cambios necesarios al producto que estamos desarrollando y genera ideas con el fin de prestar un mejor servicio y obtener un mayor posicionamiento" (Candamil; Guevara, 2008).
El benchmarking no es una técnica evaluativa pero requiere de una guía de análisis bien estructurada, integrada por un conjunto de parámetros verificables. Para la creación de esta guía en el contexto del presente trabajo se tomaron como referencia diversas propuestas, la mayoría evaluativas, de la bibliografía consultada. Entre éstas se encuentran el anexo de comparaciones de sitios web de la guía del Gobierno de Chile (2004), los indicadores relacionados con la Al en la prensa digital propuestos por Reyes (2005), el estudio de usabilidad de las páginas de inicio de los principales sitios web periodísticos españoles de García (2004), un análisis sobre la experiencia de los usuarios en medios digitales mexicanos de la Universidad de Monterrey (2009) y algunos indicadores relacionados con la IA identificados por McIntyre (2009) para seleccionar los 25 mejores sitios web de Estados Unidos (EUA).

También se tuvieron en cuenta los indicadores para la evaluación de las hemerotecas propuestos por Guallar y Abadal (2009), cuyos aspectos relacionados con la presentación de los resultados de la búsqueda resultaron útiles para la investigación, teniendo en cuenta que la búsqueda es uno de los principales servicios de los medios online.

Los indicadores generales y específicos para evaluar de forma eficiente los sitios web periodísticos propuestos por Rodríguez-Martínez, Codina y Pedraza-Jiménez (2010) constituyeron un referente primordial. De su análisis se consideraron indicadores relacionados con la influencia de la web social en la IA.

Se valoraron y se tuvieron en cuenta además otras contribuciones no enfocadas directamente al periodismo digital, así como las pautas de accesibilidad, esencialmente las de primer nivel.

Tras la revisión bibliográfica, la guía de benchmarking quedó constituida por los siguientes parámetros e indicadores:

1. Información general: aspectos de caracterización general del sistema:

- nombre y url de la web;

- país de origen;

- idioma;

- breve caracterización del sitio;

- página principal.

\section{Organización de la información}

2.1. Esquemas formados por varios grupos de contenidos, que generalmente conforman un menú o barra de navegación:

- número de esquemas empleados;

- tipos;

- número de elementos en los esquemas;

- consistencia y jerarquía visual de la información;

- clara identificación del esquema de organización de la información principal; 
- temas más comunes en los esquemas principales (grado de coincidencia en los diferentes sitios examinados);

- subesquemas de organización de la información;

- prestaciones.

2.2. Estructuras de organización de la información: formas de organizar conjuntos homogéneos de contenidos, como noticias de una sección o imágenes en una galería. Pueden ser lineales, jerárquicas, facetadas o de base de datos, entre otras.

- tipo de estructuras empleadas;

- estructuras usadas con mayor frecuencia;

- estructuras alternativas para la búsqueda de información.

\subsection{Otros aspectos de la organización de la información:}

- información que complementa las noticias;

- control del número de enlaces en las noticias.

3. Sistemas de navegación: "compuestos por los enlaces a las diferentes secciones de un sitio web, permiten ubicarnos y desplazarnos por las estructuras de la información, facilitando a los usuarios saber en cada momento dónde están, dónde pueden ir y cómo está organizada la información" (Linares, 2004). Pueden ser globales, propios de una sección o contextuales, entre otros.

- tipos de sistemas de navegación empleados;

- elementos de apoyo a los sistemas de navegación;

- indicación del estado de los enlaces;

- control de la profundidad de la navegación;

- elementos de contexto y orientación;

- enlace a la página principal.

4. Sistema de etiquetado y metadatos: conjunto de etiquetas que permiten representar los contenidos. Pueden ser textuales e icónicas y generalmente constituyen puntos de interacción. También se incluyen aquí los metadatos que describen e identifican al sitio.

- tipos de etiquetas;

- etiquetas descriptivas;

- etiquetas de navegación más comunes;

- sistema de metadatos, elementos usados;

- consistencia de los metadatos en los diferentes niveles de descripción;

- utilización de algún formato estándar de metadatos.

5. Sistema de búsqueda: diseñado para recuperar grandes volúmenes de información (el archivo de un periódico digital) o como complemento de los sistemas de navegación en el caso del buscador interno.

- posibilidades de búsqueda (simple, avanzada, contextual);

- opciones de la búsqueda avanzada;

- elementos que conforman los resultados de búsqueda;

- información en los resultados obtenidos.

6. Utilización de multimedia: complementan los contenidos aportándoles mayor riqueza y contribuyen a atraer la atención del usuario tanto en el aspecto cognitivo como en el afectivo. Permiten la optimización de la comunicación ofreciendo al usuario la posibilidad de interactuar con el contenido a partir del principio de economía reduciendo el esfuerzo de aprendizaje.

- tipo de documento multimedia;

- elementos dinámicos;

- información sobre los elementos multimedia;

- banners y elementos publicitarios;

- textos alternativos;

- metadatos de los elementos multimedia.

7. Participación, colaboración y aprendizaje: presupone el rol activo del usuario, el conocimiento se comparte y se legitima a partir de la síntesis de lo aportado por una comunidad dada, casi siempre dentro de los límites de ésta. Tiende a reunir a usuarios cuyos intereses y experiencias son similares.

- espacios para la participación;

- acciones interactivas y mecanismos de participación;

- contenidos creados por los usuarios;

- alfabetización para la participación;

- disponibilidad de herramientas sociales para compartir los contenidos.

8. Servicios y prestaciones: permiten amplificar y diversificar el uso de la información que proporciona el sitio, facilitando a los usuarios mecanismos para su gestión: enviar por correo, imprimir, compartir, etc.

- servicios más comunes;

- prestaciones frecuentes.

9. Otros aspectos observados: elementos relevantes que aunque particulares, pueden resultar novedosos y/o efectivos en la organización de la información.

\section{Selección de la muestra}

Constituyó un proceso complejo debido a la abundancia y variedad de diarios digitales existentes. Resultó difícil establecer una muestra representativa de la heterogeneidad de webs periodísticas y de los rasgos de actualización de sus sistemas de organización de la información. Para sortear esta dificultad se tuvieron en cuenta fundamentalmente indicaciones de expertos en $\mathrm{Al}$, cuyas propuestas se cruzaron con los resultados que ofrecen algunos rankings de calidad de las webs periodísticas, en función de parámetros de organización de la información. Al mismo tiempo se intentó mantener en la medida de lo posible cierta representatividad geográfica.

Resultó determinante, por tanto, el criterio de un conjunto de expertos y profesionales de Al, diseño de interacción y otras disciplinas relacionadas, quienes desde la lista de discusión Cadius opinaron sobre sitios web periodísticos que debían citarse como referentes en organización de la información. Se obtuvo la opinión de 12 expertos ( 23 de febrero de 2010), quienes respondieron de forma crítica a la pregunta: ¿cuáles cree que son los mejores exponentes de las nuevas tendencias en cuanto a organización de la información en el periodismo digital?

Se recuperaron también varios rankings elaborados con criterios de popularidad, diseño implementado, utilización de servicios de la web 2.0, tráfico diario y noticias cubiertas, 
como News top 20, International media, Newsknife y Best news website.

http://news.nettop20.com/

http://www.4imn.com/es/

http://www.newsknife.com/

http://bestuff.com/category/news-website

Los primeros medios seleccionados fueron La información. com de España y The guardian del Reino Unido, los dos más recomendados en la lista Cadius. The guardian además presentaba un número elevado de coincidencias entre las propuestas de expertos y los resultados de los rankings consultados. El número mayor de coincidencias lo tuvo The New York times, seguido por The guardian y en tercer lugar Times online.

Por otra parte, el trabajo ya mencionado de Rodríguez-Martínez, Codina y Pedraza-Jiménez (2010) destaca los sitios web de The guardian, The New York times, El mundo y El país, y señala asimismo que El mundo posee los mejores resultados en España, lo cual condujo definitivamente a seleccionar este último. Finalmente $E$ I universal de México y $L a$ nación de Argentina fueron seleccionados para que hubiera representación de América Latina.

Finalmente la muestra fue:

The guardian, Reino Unido

http://www.guardian.co.uk

The New York times, EUA

http://www.global.nytimes.com

El mundo, España

http://www.elmundo.es

La nación, Argentina

http://www.lanacion.com.ar

La información, España

http://www.lainformacion.com

El universal, México

http://www.eluniversal.com.mx

A pesar de los esfuerzos por acceder a una muestra variada y ajustada a la investigación, hay que precisar que la selección propuesta no es representativa desde una perspectiva cuantitativa y/o geográfica. Los medios elegidos responden a criterios de orden cualitativo en función de la aproximación al objeto de estudio y del propio proceso de construcción que implicó la investigación en sentido general. De ahí se desprende el predominio de medios online en idioma español.

La técnica de investigación empleada fue la observación, con matices participativos, debido a la necesidad de introducirnos en los servicios de participación de estos sitios para entender sus dinámicas. La observación y recogida de datos se efectuó durante los meses de marzo, abril y mayo de 2010 .

\section{Resultados}

El análisis permitió conocer particularidades en la organización de la información en los diarios estudiados, identificar tendencias y verificar algunas consideraciones ofrecidas por los autores citados.
Se constató una preferencia en el uso de tres esquemas de organización de la información de carácter global (se hallan en todas las webs): uno tiene una finalidad meramente noticiosa que se corresponde en mayor o menor medida con las secciones propias de un periódico tradicional, otro de participación y valor agregado, y un tercero de menor relevancia con información corporativa, referencial y de contacto.

Se observan tres esquemas de representación de la información: noticias, participación e información corporativa

Los esquemas se encuentran consistentemente ubicados en las mismas zonas en casi todas las webs. En la cabecera se localizan los esquemas principales (horizontalmente) y en el área de contenidos la mayoría de los subesquemas, específicamente en el lateral derecho. De esta forma se aprovechan las zonas de alta jerarquía visual.

La mayoría de los esquemas y estructuras están en correspondencia con el criterio de actualización continua e impacto de la noticia, lo cual hace posible una organización de los contenidos con pocos niveles de profundidad y esquemas sin mucha ambigüedad, aunque sin contrarrestar la sobrecarga de información a la que están sometidas las páginas principales. Sin embargo, al ser la actualidad y el impacto los dos criterios esenciales para organizar la información, la mayoría de estos sitios carecen de estructuras alternativas a la lineal, como por ejemplo la facética.

Se proveen nubes de etiquetas determinadas por el propio sistema y no como producto de un proceso de etiquetado social, aunque algunas son resultado de las acciones de los usuarios como es el caso de "lo más buscado".

Otra tendencia observada fue la organización de la información según el análisis del comportamiento y las rutas de navegación de los usuarios, aunque éstas también se muestran generalmente de forma lineal.

A pesar de una participación y colaboración cada vez más frecuente en estos sistemas, se utiliza sólo el resultado de las acciones y no criterios organizativos de los usuarios, como sucede en los marcadores y redes sociales. Por ejemplo, no se pueden etiquetar las noticias, por lo tanto no se utilizan estructuras folksonómicas.

La nación presenta gráficamente las noticias más leídas basándose en las redes sociales

Una observación interesante es la forma particular de $L a$ nación de presentar gráficamente las noticias más leídas basándose en las redes sociales (figura 1), lo que constituye una novedad para el periodismo digital, que se ha puesto en marcha también en otros medios.

Es relativamente novedosa la visualización de estructuras (tabla 1) basadas en clústeres de noticias relacionadas, re- 


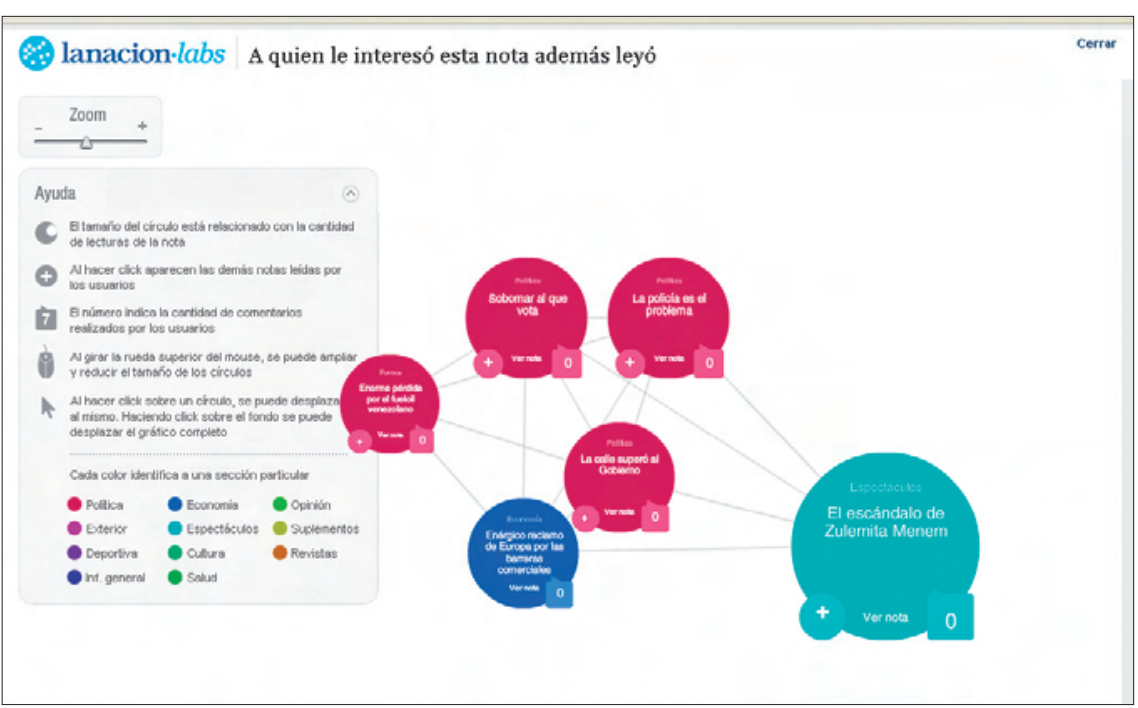

Figura 1. Uso de las rutas de navegación de los usuarios en La nación

sultado también del análisis estadístico de las acciones de los usuarios según varios criterios (más leído, más comentado, más enviado, etc.).

Los metadatos (tabla 2) son utilizados por todos los diarios, lo cual demuestra su importancia para el posicionamiento

\begin{tabular}{|l|c|c|c|c|c|c|}
\hline $\begin{array}{l}\text { Estructura/ } \\
\text { Sitio }\end{array}$ & $\begin{array}{c}\text { The } \\
\text { guar- } \\
\text { dian }\end{array}$ & $\begin{array}{c}\text { The New } \\
\text { york } \\
\text { times }\end{array}$ & $\begin{array}{c}\text { EI } \\
\text { mundo }\end{array}$ & $\begin{array}{c}\text { La } \\
\text { nación }\end{array}$ & $\begin{array}{c}\text { La } \\
\text { informa- } \\
\text { ción }\end{array}$ & $\begin{array}{c}\text { EI } \\
\text { univer- } \\
\text { sal }\end{array}$ \\
\hline Cronológica & $\mathrm{x}$ & $\mathrm{x}$ & $\mathrm{x}$ & $\mathrm{x}$ & $\mathrm{x}$ & $\mathrm{x}$ \\
\hline $\begin{array}{l}\text { Por } \\
\text { relevancia e } \\
\text { impacto }\end{array}$ & $\mathrm{x}$ & $\mathrm{x}$ & $\mathrm{x}$ & $\mathrm{x}$ & $\mathrm{x}$ & $\mathrm{x}$ \\
\hline $\begin{array}{l}\text { Criterios } \\
\text { estadísticos } \\
\text { simples }\end{array}$ & $\mathrm{x}$ & $\mathrm{x}$ & $\mathrm{x}$ & $\mathrm{x}$ & $\mathrm{x}$ & $\mathrm{x}$ \\
\hline Clústeres & $\mathrm{x}$ & & $\mathrm{x}$ & $\mathrm{x}$ & & \\
\hline Alfabética & $\mathrm{x}$ & & & $\mathrm{x}$ & & \\
\hline $\begin{array}{l}\text { Nube de } \\
\text { etiquetas }\end{array}$ & $\mathrm{x}$ & & & $\mathrm{x}$ & & \\
\hline $\begin{array}{l}\text { Redes } \\
\text { sociales }\end{array}$ & & & & $\mathrm{x}$ & & \\
\hline
\end{tabular}

Tabla 1. Estructuras de organización de la información más empleadas

\begin{tabular}{|c|c|c|c|c|c|c|}
\hline Título / Sitio & $\begin{array}{l}\text { The } \\
\text { guar- } \\
\text { dian }\end{array}$ & $\begin{array}{c}\text { The New } \\
\text { York } \\
\text { times }\end{array}$ & $\begin{array}{c}\text { El } \\
\text { mundo }\end{array}$ & $\begin{array}{c}\text { La } \\
\text { nación }\end{array}$ & $\begin{array}{c}\text { La } \\
\text { informa- } \\
\text { ción }\end{array}$ & $\begin{array}{c}\text { EI } \\
\text { univer- } \\
\text { sal }\end{array}$ \\
\hline Título & $x$ & $x$ & $x$ & $x$ & $x$ & $x$ \\
\hline Descripción & $x$ & $x$ & $x$ & $x$ & $x$ & $x$ \\
\hline $\begin{array}{l}\text { Palabras } \\
\text { clave }\end{array}$ & $x$ & $x$ & $x$ & $\mathrm{x}$ & $x$ & $x$ \\
\hline Autor & & & $x$ & & $x$ & $x$ \\
\hline Robots & & $x$ & & & $x$ & $x$ \\
\hline $\begin{array}{l}\text { Organiza- } \\
\text { ción }\end{array}$ & & & $x$ & & $x$ & \\
\hline Verificación & & & & $x$ & $x$ & \\
\hline Fecha & $x$ & & & & & \\
\hline Idioma & & & $x$ & & & \\
\hline Copyright & & & & $x$ & & \\
\hline Distribución & & & & & $x$ & \\
\hline $\begin{array}{l}\text { Tipo de } \\
\text { recurso }\end{array}$ & & & & & $x$ & \\
\hline
\end{tabular}

Tabla 2. Metadatos más usados web y la búsqueda interna, si bien no son usados óptimamente en sus diferentes niveles de descripción (página principal / sección / artículo), y tampoco es frecuente el uso de estándares específicos de metadatos.

Los sistemas de búsqueda están bien concebidos y estructurados. Ofrecen la posibilidad tanto de búsqueda global como contextual o por secciones, con varias opciones de búsqueda y filtrado, añadiendo información presente en los comentarios y blogs, y mostrando elementos de valor añadido como por ejemplo las búsquedas más populares.

Los resultados se ordenan siempre cronológicamente con la alternativa de agruparlos por relevancia. Se puede refinar la búsqueda por fechas específicas, tipo de documento, sección y fuente o publicación (tabla 3).

Con respecto a la utilización de los elementos multimedia se puede identificar el cumplimiento de algunas pautas de accesibilidad, entre ellas el control sobre los elementos dinámicos y cambiantes y la utilización de textos alternativos.

La búsqueda está bien concebida y estructurada, ofreciendo las posibilidades global, contextual o por secciones

\begin{tabular}{|l|c|c|c|c|c|c|}
\hline $\begin{array}{c}\text { Elemento / } \\
\text { Sitio }\end{array}$ & $\begin{array}{c}\text { The } \\
\text { guar- } \\
\text { dian }\end{array}$ & $\begin{array}{c}\text { The New } \\
\text { York } \\
\text { times }\end{array}$ & $\begin{array}{c}\text { EI } \\
\text { mundo }\end{array}$ & $\begin{array}{c}\text { La } \\
\text { nación }\end{array}$ & $\begin{array}{c}\text { La } \\
\text { informa- } \\
\text { ción }\end{array}$ & $\begin{array}{c}\text { EI } \\
\text { univer- } \\
\text { sal }\end{array}$ \\
\hline $\begin{array}{l}\text { Indicación } \\
\text { del término } \\
\text { buscado }\end{array}$ & $\mathrm{x}$ & $\mathrm{x}$ & $\mathrm{x}$ & $\mathrm{x}$ & $\mathrm{x}$ & $\mathrm{x}$ \\
\hline $\begin{array}{l}\text { Término } \\
\text { señalado } \\
\text { en los } \\
\text { resultados }\end{array}$ & $\mathrm{x}$ & $\mathrm{x}$ & $\mathrm{x}$ & $\mathrm{x}$ & & \\
\hline $\begin{array}{l}\text { Número de } \\
\text { resultados }\end{array}$ & $\mathrm{x}$ & $\mathrm{x}$ & $\mathrm{x}$ & $\mathrm{x}$ & $\mathrm{x}$ & $\mathrm{x}$ \\
\hline $\begin{array}{l}\text { Número de } \\
\text { páginas de } \\
\text { resultados }\end{array}$ & & $\mathrm{x}$ & $\mathrm{x}$ & $\mathrm{x}$ & $\mathrm{x}$ & $\mathrm{x}$ \\
\hline $\begin{array}{l}\text { Filtrar los } \\
\text { resultados }\end{array}$ & $\mathrm{x}$ & $\mathrm{x}$ & $\mathrm{x}$ & $\mathrm{x}$ & & \\
\hline $\begin{array}{l}\text { Términos de } \\
\text { búsqueda } \\
\text { relacionados }\end{array}$ & $\mathrm{x}$ & $\mathrm{x}$ & $\mathrm{x}$ & $\mathrm{x}$ & & \\
\hline $\begin{array}{l}\text { Retroali- } \\
\text { mentación } \\
\text { sobre la } \\
\text { búsqueda }\end{array}$ & $\mathrm{x}$ & $\mathrm{x}$ & $\mathrm{x}$ & $\mathrm{x}$ & \\
\hline $\begin{array}{l}\text { Búsquedas } \\
\text { más } \\
\text { populares }\end{array}$ & & $\mathrm{x}$ & $\mathrm{x}$ & \\
\hline $\begin{array}{l}\text { Ayuda en la } \\
\text { búsqueda }\end{array}$ & $\mathrm{x}$ & $\mathrm{x}$ & & \\
\hline
\end{tabular}

Tabla 3. Elementos en las páginas de los resultados (incluyendo las opciones de la búsqueda avanzada) 
Está extendido el uso de estos últimos para proporcionar información acerca de archivos icónicos y audiovisuales, aunque la mayoría de las veces no describen sino reproducen el pie de foto o el titular de la noticia. Además la asignación de metadatos a estos materiales no es sistemática.

La participación y la colaboración (tabla 4) constituyen elementos representativos. Aunque no se hace un uso intensivo de las iniciativas y mecanismos que existen para ello, se dedican secciones y subsecciones a este propósito ("Soy corresponsal" en La nación, "Reportero ciudadano" y "Voz del lector" en El universal), donde podemos obtener una identidad, crear un perfil, personalizar la interfaz y ser reconocidos socialmente por nuestro comportamiento. Asimismo disponen de espacios de alfabetización para garantizar una óptima participación.

También se vinculan los contenidos de estos sitios a marcadores y redes de la web social donde Twitter y Facebook son los más usados.

\begin{tabular}{|c|c|c|c|c|c|c|}
\hline $\begin{array}{c}\text { Acciones / } \\
\text { Sitio }\end{array}$ & $\begin{array}{l}\text { The } \\
\text { guar- } \\
\text { dian }\end{array}$ & $\begin{array}{c}\text { The New } \\
\text { York } \\
\text { times }\end{array}$ & $\begin{array}{c}\text { El } \\
\text { mundo }\end{array}$ & $\begin{array}{c}\text { La } \\
\text { nación }\end{array}$ & $\begin{array}{c}\text { La } \\
\text { informa- } \\
\text { ción }\end{array}$ & $\begin{array}{c}\text { El } \\
\text { univer- } \\
\text { sal }\end{array}$ \\
\hline $\begin{array}{l}\text { Comentar } \\
\text { las noticias }\end{array}$ & $x$ & $x$ & $x$ & $x$ & $x$ & $x$ \\
\hline $\begin{array}{l}\text { Compartir } \\
\text { las noticias } \\
\text { en redes } \\
\text { sociales }\end{array}$ & $x$ & $x$ & $x$ & $x$ & $x$ & $x$ \\
\hline $\begin{array}{l}\text { Participa- } \\
\text { ción en } \\
\text { encuestas, } \\
\text { votaciones, } \\
\text { foros y } \\
\text { debates }\end{array}$ & $x$ & $x$ & $x$ & $x$ & $x$ & \\
\hline $\begin{array}{l}\text { Incorporar } \\
\text { noticias, } \\
\text { fotos, suge- } \\
\text { rencias }\end{array}$ & $x$ & $x$ & $x$ & $x$ & & $x$ \\
\hline $\begin{array}{l}\text { Registrarse y } \\
\text { personalizar } \\
\text { la interfaz }\end{array}$ & $x$ & $x$ & $x$ & $x$ & $x$ & \\
\hline $\begin{array}{l}\text { Votar y } \\
\text { valorar las } \\
\text { noticias }\end{array}$ & & & $x$ & $x$ & & \\
\hline $\begin{array}{l}\text { Rectificar las } \\
\text { noticias }\end{array}$ & & & $x$ & & $x$ & \\
\hline
\end{tabular}

Tabla 4. Acciones interactivas y de participación

\section{La participación de los usuarios se con-} cibe como opinión y no aportación de contenido

Los sitios estudiados pueden considerarse destacados a nivel general en las prácticas relativas al periodismo digital y la IA. Sin embargo, la participación se concibe mayoritariamente desde el punto de vista de la opinión del usuario y no de la aportación de contenidos, por lo que queda limitada a espacios específicos y no se integra en la amplia gama de informaciones que presentan los diarios. Por tanto, se considera que las webs de prensa necesitarían reconocer y aprobar los contenidos con fines informativos creados mediante las herramientas sociales e incorporarlos a su proceso de producción periodística sin dejar de atender a su función comunicacional.

\section{Conclusiones}

La organización de la información en el periodismo digital y su evaluación han sido poco estudiadas, considerándose sólo y en el mejor de los casos, como un parámetro o un conjunto de indicadores en la evaluación integral de este tipo de sistemas. Aún así, se considera que es de gran relevancia para que la organización y navegación de los sitios web periodísticos no responda sólo a los criterios periodísticos de impacto, actualidad y posicionamiento, sino que tenga en cuenta además las diferentes experiencias de sus usuarios y por lo tanto diversidad de alternativas para acceder a la información.

Las principales tendencias que se pueden señalar a partir de este estudio son: una navegación global determinada por tres criterios esenciales: el carácter eminentemente noticioso, los servicios y el valor agregado, y la información corporativa y de contacto; el uso de criterios estadísticos sobre el comportamiento de los usuarios para facilitar la navegación y recuperación de información; el empleo de técnicas de visualización de información; los diferentes mecanismos de participación y colaboración; y la vinculación a las redes sociales.

A pesar de ello se observó que la organización de la información no alcanza en muchas ocasiones unos niveles aceptables, lo que da lugar a páginas abigarradas de información, y que la participación de los usuarios es bastante limitada.

Se pudo constatar además que el conjunto de indicadores utilizados pueden ser empleados en otros estudios con objetivo evaluativo.

\section{Bibliografía}

Candamil-Llano, Mauricio; Guevara-Hurtado, Adrián-Fernando. Análisis de sitios web universitarios colombianos mediante evaluaciones heurísticas para el proyecto del nuevo portal web Unicauca.

http://webparahumanos.wordpress.com/

http://www.slideshare.net/mauriciocandamil/mtodos-dearquitectura-de-informacin-para-la-organizacin-de-conte nidos-en-el-diseo-de-sitios-web-universitarios-unicaucae duco-un-caso-prctico

García, Juan-Carlos. "Usabilidad de las páginas de inicio de los diarios digitales españoles". Scire, 2004, v. 10, n. 2, pp. 9-31.

http://www.um.es/gtiweb/juancar/curri/scire_garciago mez_2004.pdf

García, Mario. "Is top navigation still necessary on news sites?". García interactive, 16 marzo 2009.

http://garciainteractive.com/blog/view/37/

Gobierno de Chile. Guía para el desarrollo de sitios web. Versión 1.0. 2004.

http://www.guiaweb.gob.cl/guia/index.htm

Guallar, Javier; Abadal, Ernest. "Evaluación de hemerotecas de prensa digital: indicadores y ejemplos de buenas prácti- 
cas". El profesional de la información, 2009, mayo-junio, v. 18, n. 3, pp. 255-269.

http://eprints.rclis.org/16899/1/epi09_guallar-abadal_ evaluacion_hemerotecas.pdf

Linares, Valentín. Análisis de sistemas de navegación de sitios Web, 2004.

http://www.vlinares.net/portafolio/html/uoc/iho/vlinares_ memoria.pdf

Martínez-Priego, Chema. "Herramientas metodológicas para la arquitectura de la información". Simdalom, 18 julio 2006.

http://www.simdalom.com/blog/2006/07/18/herramien tas-metodologicas-para-la-arquitectura-de-la-informacion/

Mclntyre, Douglas A. "The newspapers: rating the top 25 newspaper websites". 2417 Wall St.com, 3 junio 2008.

http://247wallst.com/2008/06/03/the-newspapers/

Purcel, Kristen; Rainie, Lee; Mitchell, Amy; Rosenstiel, Tom; Olmstead, Kenny. "Understanding the participatory news consumer". Pew internet, 1 marzo 2010.

http://pewinternet.org/Reports/2010/Online-News. aspx? $r=1$

Reyes, Livia M. “Evaluación de las publicaciones cubanas de cara a internet: experiencia de validación de una herramienta para medir su calidad". Ciencias de la información, 2005, agosto, v. 36, n. 2, pp. 3-29.

Rodríguez-Martínez, Ruth; Codina, Lluís; Pedraza-Jiménez, Rafael. "Cibermedios y web 2.0: modelo de análisis y resultados de aplicación". El profesional de la información, 2010, enero-febrero, v. 19, n. 1.

http://www.lluiscodina.com/periodismo20.pdf

Snell, Steven. "Newspaper website design: trends and examples". Smashing magazine, 2006.

Universidad de Monterrey. Estudio de la experiencia del usuario en portales de periódicos mexicanos.

http://www.periodicosenlinea.com.mx/news/resumen.html

\section{Cualquier título. Cualquier proveedor Una sola Plataforma.}
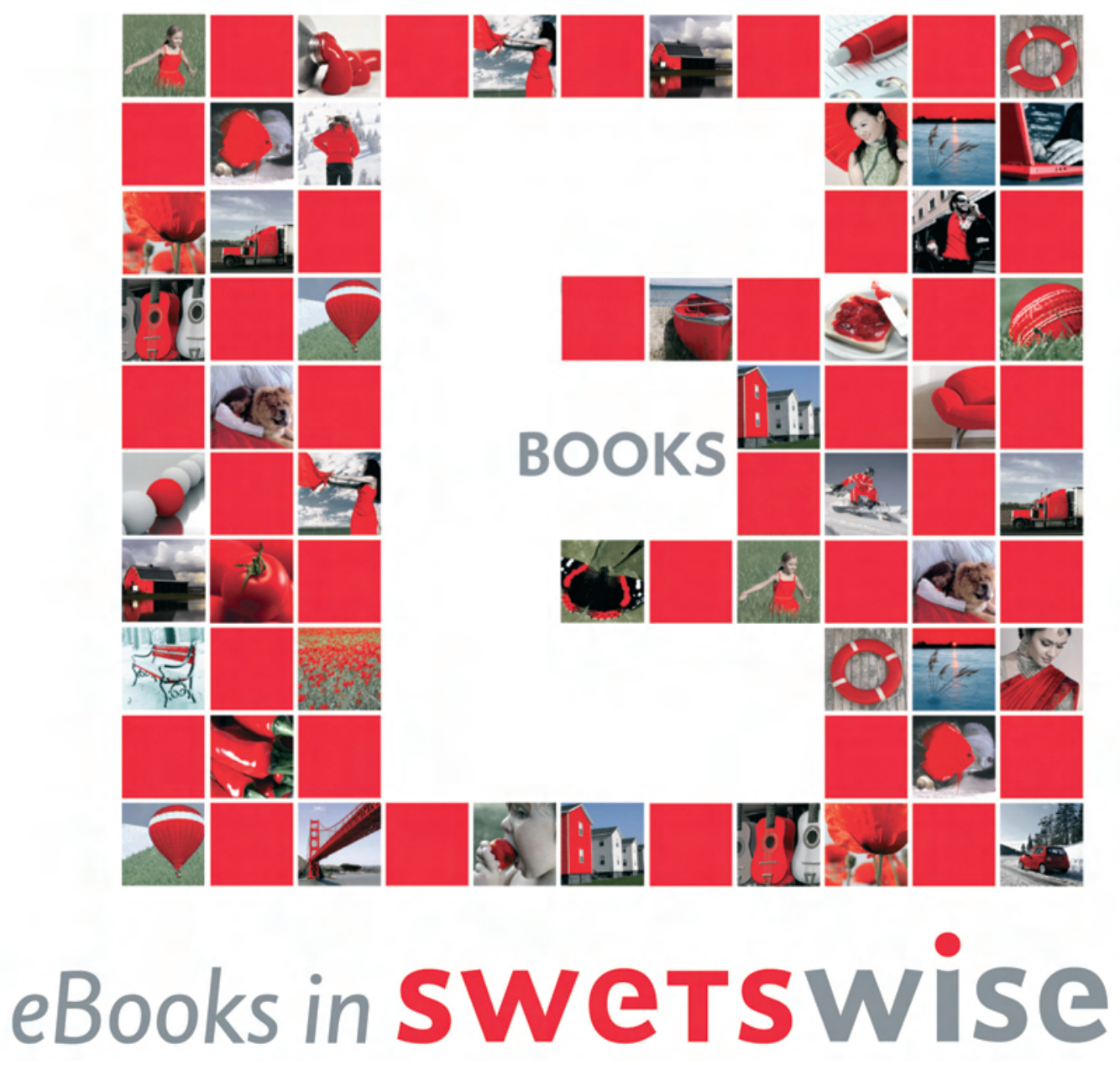